\title{
AEDGE: Atomic experiment for dark matter and gravity exploration in space
}

\begin{abstract}
Andrea Bertoldi $^{1} \cdot$ Kai Bongs $^{2} \cdot$ Philippe Bouyer $^{1}$ - Oliver Buchmueller ${ }^{3}$ (D) . Benjamin Canuel $^{1}$. Laurentiu-loan Caramete ${ }^{4}$. Maria Luisa Chiofalo ${ }^{5}$. Jonathon Coleman ${ }^{6}$. Albert De Roeck ${ }^{7,8}$. John Ellis ${ }^{9,10,11}$ • Peter W. Graham ${ }^{12}$. Martin G. Haehnelt ${ }^{13}$. Aurélien Hees ${ }^{14}$. Jason Hogan ${ }^{12}$. Wolf von Klitzing ${ }^{15}$. Markus Krutzik $^{16}$. Marek Lewicki ${ }^{9,17}$. Christopher McCabe ${ }^{9}$ Achim Peters $^{16}$. Ernst Rasel ${ }^{18}$. Albert Roura ${ }^{19}$. Dylan Sabulsky ${ }^{1}$. Stephan Schiller ${ }^{20}$. Christian Schubert $^{18}$. Carla Signorini ${ }^{5}$. Fiodor Sorrentino ${ }^{21}$. Yeshpal Singh ${ }^{2}$. Guglielmo Maria Tino 22,23 . Ville Vaskonen ${ }^{9,10} \cdot$ Ming-Sheng Zhan ${ }^{24}$
\end{abstract}

Received: 3 December 2020 / Accepted: 27 January 2021 / Published online: 12 March 2021

(C) The Author(s) 2021

\begin{abstract}
This article contains a summary of the White Paper submitted in 2019 to the ESA Voyage 2050 process, which was subsequently published in EPJ Quantum Technology (AEDGE Collaboration et al. EPJ Quant. Technol. 7,6 2020). We propose in this White Paper a concept for a space experiment using cold atoms to search for ultralight dark matter, and to detect gravitational waves in the frequency range between the most sensitive ranges of LISA and the terrestrial LIGO/Virgo/KAGRA/INDIGO experiments. This interdisciplinary experiment, called Atomic Experiment for Dark Matter and Gravity Exploration (AEDGE), will also complement other planned searches for dark matter, and exploit synergies with other gravitational wave detectors. We give examples of the extended range of sensitivity to ultra-light dark matter offered by AEDGE, and how its gravitational-wave measurements could explore the assembly of super-massive black holes, first-order phase transitions in the early universe and cosmic strings. AEDGE will be based upon technologies now being developed for terrestrial experiments using cold atoms, and will benefit from the space experience obtained with, e.g., LISA and cold atom experiments in microgravity.
\end{abstract}

Keywords Dark matter · Dark energy · Gravitational waves · Quantum technology

Oliver Buchmueller

o.buchmueller@imperial.ac.uk

Extended author information available on the last page of the article. 


\section{Preface}

This article contains a summary of the White Paper submitted in 2019 by the same authors to the ESA Voyage 2050 process, and also mentions a few subsequent scientific and technical developments. The paper was published in EPJ Quantum Technology [1], where we have welcomed as supporting authors participants in the Workshop on Atomic Experiments for Dark Matter and Gravity Exploration held at CERN [2], as well as other interested scientists.

\section{Science case}

Two of the most important issues in fundamental physics, astrophysics and cosmology are the nature of dark matter (DM) and the exploration of the gravitational wave (GW) spectrum.

Multiple observations from the dynamics of galaxies and clusters to the spectrum of the cosmological microwave background (CMB) radiation measured by ESA's Planck satellite and other [3] experiments indicate that there is far more DM than conventional matter in the Universe, but its physical composition remains a complete mystery. The two most popular classes of DM scenario invoke either coherent waves of ultra-light bosonic fields, or weakly-interacting massive particles (WIMPs). In the absence so far of any positive indications for WIMPs from accelerator and other laboratory experiments, there is increasing interest in ultra-light bosonic candidates, many of which appear in theories that address other problems in fundamental physics. Such bosons are among the priority targets for AEDGE.

The discovery of GWs by the LIGO [4] and Virgo [5] laser interferometer experiments has opened a new window on the Universe, through which waves over a wide range of frequencies can provide new information about high-energy astrophysics and cosmology. Just as astronomical observations at different wavelengths provide complementary information about electromagnetic sources, measurements of GWs in different frequency bands are complementary and synergistic. In addition to the ongoing LIGO and Virgo experiments at relatively high frequencies $\gtrsim 10 \mathrm{~Hz}$, which will soon be joined by the KAGRA [6] detector in Japan and the INDIGO project [7] to build a LIGO detector in India, with the Einstein Telescope (ET) $[8,9]$ and Cosmic Explorer (CE) [10] experiments being planned for similar frequency ranges, ESA has approved for launch before the period being considered for Voyage 2050 missions the LISA mission, which will be most sensitive at frequencies $\lesssim 10^{-1} \mathrm{~Hz}$, and the Taiji [11] and TianQin [12] missions proposed in China will have similar sensitivity to LISA. AEDGE is optimized for the mid-frequency range between LISA/Taiji/TianQin and LIGO/Virgo/KAGRA/INDIGO/ET/CE ${ }^{1}$. This range is ideal for probing the formation of the super-massive black holes known to be present in

\footnotetext{
${ }^{1}$ The ALIA proposal in Europe [13] and the DECIGO proposal in Japan [14] have been aimed at a similar frequency range, and the scientific interest of this frequency range has recently been stressed in $[15,16]$ and [17].
} 
many galaxies. Also, AEDGE's observations of astrophysical sources will complement those by other GW experiments at lower and higher frequencies, completing sets of measurements from inspiral to merger and ringdown, yielding important synergies as we illustrate below. GWs are the other priority targets for AEDGE.

In addition to these primary scientific objectives, several other potential objectives for cold atom experiments in space are under study. These may include constraining possible variations in fundamental constants, probing dark energy, and probing basic physical principles such as Lorentz invariance and quantum mechanics. Cold quantum gases provide powerful technologies that are already mature for the AEDGE goals, while also developing rapidly [18]. The developments of these technologies can be expected to offer AEDGE more possibilities on the Voyage 2050 time scale. AEDGE is a uniquely interdiscplinary and versatile mission.

\section{Experimental considerations}

The design of AEDGE requires two satellites operating along a single line-of-sight and separated by a long distance. The payload of each satellite will consist of cold atom technology as developed for state-of-the-art atom interferometry and atomic clocks. As two satellites are needed to accomplish its science goals, the AEDGE mission planning costs are estimated to be in the range of an L-class mission. However, in view of the international interest in the AEDGE science goals, the possibility of international cooperation and co-funding of the mission may be investigated.

There are several cold atom projects based on various technologies that are currently under construction, planned or proposed, which address the principal technical challenges and could be considered in a detailed design for a mission proposal and corresponding satellite payload. However, all of these options require the same basic detector and mission configuration outlined above. For the option presented in the White Paper we chose to base our discussion on the concept outlined in in [19-22], which is currently the most advanced design for a space mission (Fig. 1).

This concept links clouds of cold atomic strontium in a pair of satellites in medium earth orbit (MEO) via pulsed continuous-wave lasers that induce the $698 \mathrm{~nm}$ atomic clock transition, and detect momentum transfers from the electromagnetic field to the strontium atoms, which act as test masses in a double atom interferometer scheme.

\section{Technological readiness}

AEDGE will benefit from the experience gained with LISA Pathfinder in free-fall control and LISA itself in operating laser interferometers over large distances. We have identified the following three additional high-level technical requirements that are critical for AEDGE:

- Demonstrate reliable functioning of atom interferometry on a large terrestrial scale $\gtrsim 100 \mathrm{~m}$; 


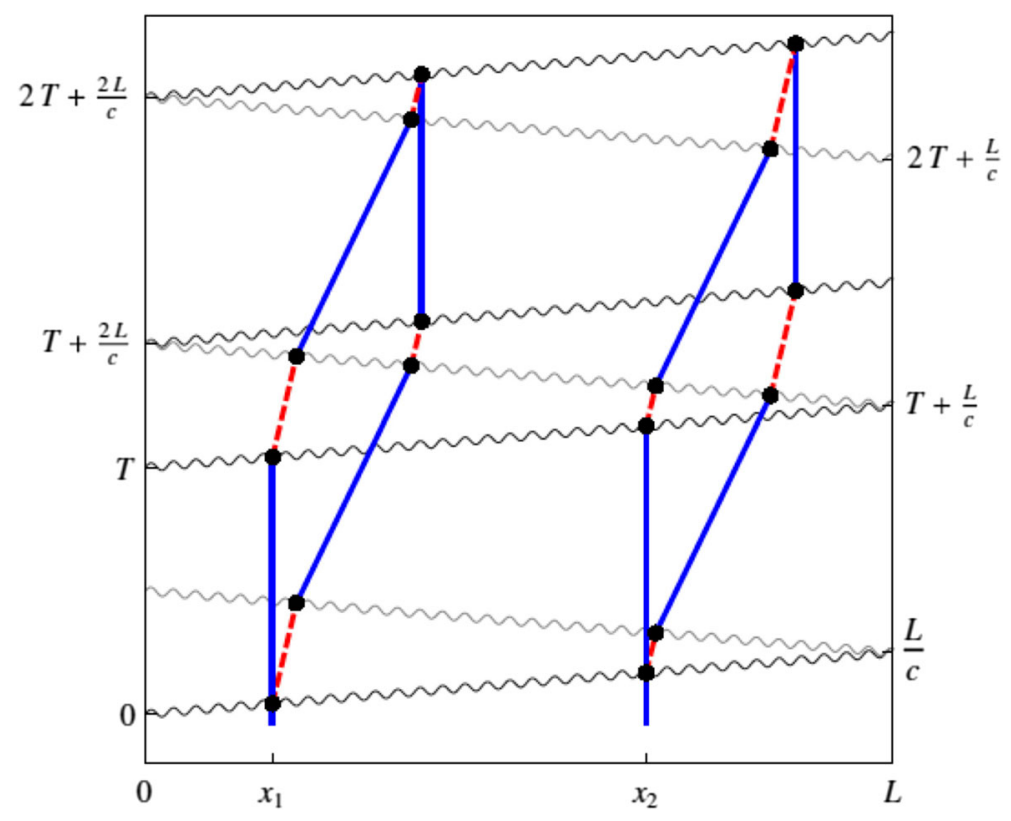

Fig. 1 Space-time diagram of the operation of a pair of cold-atom interferometers based on single-photon transitions between the ground state (blue) and the excited state (red dashed). The laser pulses (wavy lines) travelling across the baseline from opposite sides are used to divide, redirect, and recombine the atomic de Broglie waves, yielding interference patterns that are sensitive to the modulation of the light travel time caused by DM or GWs (from [19]). For clarity, the sizes of the atom interferometers are shown on an exaggerated scale

- Demonstrate that the design parameters assumed here, such as the LMT enhancement, phase noise control, interrogation time, etc., can be achieved;

- Demonstrate the robustness of cold atom technology in the space environment.

Several terrestrial atom interferometer projects that would serve as demonstrators for different technologies are under construction, planned or proposed.

These include three large-scale prototype projects at the $100-\mathrm{m}$ scale that are funded and currently under construction, namely MAGIS-100 in the US [21], MIGA in France [23], ZAIGA in China [24] and now the first stage of AION in the UK [25], and there are projects to build one or several more km-scale detectors in the US (at the Sanford Underground Research facility, SURF), in Europe (MAGIAadvanced [26, 27], ELGAR [28]) and in China (advanced ZAIGA) that would serve as the ultimate technology readiness demonstrators for AEDGE. In parallel to these large-scale prototype projects, several other cold atom projects are in progress or planned, demonstrating the general readiness of the technology including the scaling of the basic parameters that are required for AEDGE.

Moreover, several cold atom experiments (CACES [29], MAIUS [30], CAL [31]) and underlying optical key technologies (FOKUS [32], KALEXUS [33], JOKARUS [34]) have already demonstrated reliable operation in space, and much more experience will be gained in the coming years. 
In addition there are ongoing NASA, Chinese, ESA, German and French projects to conduct cold atom experiments in space, some of which have already provided operational experience with cold atoms in space or microgravity environments. These include the Cold Atom Laboratory (CAL) experiment on the ISS [31], the Chinese Atomic Clock Ensemble in Space (CACES) that has demonstrated in-orbit operation of an atomic clock based on laser-cooled rubidium atoms [29], the Atomic Clock Ensemble in Space (ACES/PHARAO) project led by ESA plans to install ultra-stable atomic cesium clocks on the ISS [35, 36], the Bose-Einstein Condensate and Cold Atom Laboratory (BECCAL) project [37], the ICE experiment that recently reported the all-optical formation of a BEC in the microgravity environment obtained on an Einstein elevator [38], and the ISS Space Optical Clock (I-SOC) project of ESA [39, 40] to use cold strontium atoms in space to compare and synchronize atomic clocks worldwide.

Other proposals for atomic experiments in space to probe fundamental physics include STE-QUEST [41], the Space Atomic Gravity Explorer (SAGE) mission [22], the SagnAc interferometer for Gravitational wavE proposal (also called SAGE) [42] and the Atomic Interferometric Gravitational-Wave Space Observatory (AIGSO) proposed in China [43].

\section{Summary}

The nature of DM is one of the most important and pressing in particle physics and cosmology, and one of the favoured possibilities is that it is provided by coherent waves of some ultra-light boson. AEDGE will be able to explore large ranges of the parameter spaces of such models, complementing the capabilities of other experiments.

Experience with electromagnetic waves shows the advantages of making astronomical observations in a range of different frequencies, and the same is expected to hold in the era of gravitational astronomy. There are advanced projects to explore the GW spectrum with maximum sensitivities at frequencies $\gtrsim 10 \mathrm{~Hz}$ and below $\lesssim 10^{-2} \mathrm{~Hz}$, but no approved project has peak sensitivity in the mid-frequency band between them. AEDGE would be ideal for exploiting the scientific opportunities in the mid-frequency band, complementing other experiments and offering synergies with them.

Other possible opportunities for AEDGE in fundamental physics, astrophysics and cosmology have been identified, but not yet explored in detail.

The roadmap towards the AEDGE mission includes the following elements:

- Today to 2025: Prototype 10-m facilities in the US, Europe and China, being extended to $\mathcal{O}(100) \mathrm{m}$;

- 2025 to 2035: scaling of 100-m facilities to km-scale infrastructures;

- These experiments will demonstrate the reliability of cold-atom interferometers capable of achieving or surpassing the technical requirements for AEDGE;

- Operation of LISA will demonstrate the operation of large-scale laser interferometry in space; 
- In parallel, a vigorous technology development programme should be set up, pursued and coordinated on a European-wide level in order to maximize efficiency and avoid duplication.

AEDGE is a uniquely interdisciplinary mission that will harness cold atom technologies to address key issues in fundamental physics, astrophysics and cosmology that can be realized within the Voyage 2050 Science Programme of ESA. The worldwide spread of the authors of this article indicate that there could be global interest in participating in this mission.

\section{Recent developments}

Following submission of this White Paper, an expanded version with over 100 additional authors was published [1]. This contains a number of refinements of the science arguments, notably a discussion of the synergies to be obtained by including AEDGE in a network of complementary detectors including LISA, LIGO, Virgo et al. The expanded version also stresses the abilities of AEDGE to measure and localize prospective mergers during their early infall phases and predict accurately the times before the mergers themselves, which are of great interest for multi-messenger astronomy. These AEDGE measurements would also provide precise tests of general relativity, e.g., by constraining the gravitino mass [44].

An interesting and exciting new technical development is the possibility of doing data analysis and alert generation on-board using neural networks. On-board data processing can become quite important for overcoming the massive limitations on the speed of data transfer, in order to generate alerts for space or Earth observatories in the context of multi-messenger observations. The proposed solution, making onboard data analysis and processing on space-qualified FPGAs using neural networks, has been already presented to ESA, and its implementation for future missions has been encouraged.

As mentioned in the White Paper, there is an urgent need to raise the Technology Readiness Level (TRL) of cold atom devices for space applications, including but not limited to interferometers. We note in this connection the recent successful observation of Bose-Einstein condensation on the ISS by the BEC Collaboration [45], as well as recent progress in the development of technologies for quantum communication [46].

Finally, we also note that the proposal for the AION ground-based atom interferometer project [25] has recently been approved in the UK, joining the MAGIS project in the US, the MIGA project in France, and the ZAIGA project in China, which had been approved previously.

\footnotetext{
Abbreviations ACES/PHARAO, Atomic Clock Ensemble in Space/Projet d'Horloge à Refroidissement d'Atomes en Orbite; AIGSO, Atomic Interferometric Gravitational-Wave Space Observatory; ALIA, Advanced Laser Interferometer Antenna; ALP, Axion-like particle; BEC, Bose-Einsten Condensate; BECCAL, Bose-Einstein Condensate and Cold Atom Laboratory; BS, Beam splitter; CACES, Cold Atom Clock Experiment in Space; CAL, Cold Atom Laboratory; CERN, Conseil Européen pour le Recherche Nucléaire; CMB, Cosmic Microwave Background; DECIGO, Deci-Hertz Interferometer Gravitational
} 
Wave Observatory; DLR, Deutsches Zentrum für Luft- und Raumfahrt; DM, Dark Matter; ESA, European Space Agency; FOKUS, Faserlaserbasierter Optischer Kammgenerator unter Schwerelosigkeit; INFN, Istituto Nazionale per la Fisica Nucleare; ICE, atomique à sources Cohérentes pour l'Espace; ISO, International Organization for Standardization; I-SOC, ISS space optical clock; ISS, International Space Station; JOKARUS, Jod Kamm Resonator unter Schwerelosigkeit; KALEXUS, Kalium-Laserexperimente unter Schwerelosigkeit; LMT, Large momentum transfer; MAGIA, Misura Accurata di G mediante Interferometria Atomica; MAIUS, Materiewellen Interferometer Unter Schwerelosigkeit; MEO, Medium earth orbit; MICROSCOPE, Micro-Satellite à traînée Compensée pour l'Observation du Principe d'Equivalence; NASA, National Aeronautics and Space Agency; QUANTUS, Quantengase Unter Schwerelosigkeit; SAGAS, Search for Anomalous Gravitation using Atomic Sensors; SAGE, Space Atomic Gravity Explorer, Sagnac interferometer for gravitational wave; SAI, Space Atom Interferometer; STE-QUEST, Space-Time Explorer and QUantum Equivalence Principle Space Test; SURF, Sanford underground research facility; TRL, Technology readiness level; TTM, Tip-tilt mirror; WIMP, Weakly-Interacting Massive Particle —.

Acknowledgments We thank CERN for kindly hosting the workshop where the concept for this proposed experiment was developed.

Author Contributions The author Oliver Buchmueller is the contact person for this paper. The following authors are the original proponents of a submission in response to the Call for White Papers for the Voyage 2050 long-term plan in the ESA Science Programme: Andrea Bertoldi, Kai Bongs, Philippe Bouyer, Oliver Buchmueller, Benjamin Canuel, Laurentiu-Ioan Caramete, Maria Luisa Chiofalo, Jonathon Coleman, Albert De Roeck, John Ellis, Peter W. Graham, Martin G. Haehnelt, Aurelien Hees, Jason Hogan, Wolf von Klitzing, Markus Krutzik, Marek Lewicki, Chris McCabe, Achim Peters, Ernst Rasel, Albert Roura, D. O. Sabulsky, Stephan Schiller, Christian Schubert, Carla Signorini, Fiodor Sorrentino, Yeshpal Singh, Guglielmo Tino, Ville Vaskonen, Ming-Sheng Zhan. All authors discussed the content of the paper and contributed to the writing of the manuscript. All authors read and approved the final manuscript.

Funding The project proposed in this paper is not yet funded.

Availability of Data and Materials For all requests relating to the paper, please contact author Oliver Buchmueller.

\section{Declarations}

Conflict of Interests The authors declare that they have no competing interests.

Open Access This article is licensed under a Creative Commons Attribution 4.0 International License, which permits use, sharing, adaptation, distribution and reproduction in any medium or format, as long as you give appropriate credit to the original author(s) and the source, provide a link to the Creative Commons licence, and indicate if changes were made. The images or other third party material in this article are included in the article's Creative Commons licence, unless indicated otherwise in a credit line to the material. If material is not included in the article's Creative Commons licence and your intended use is not permitted by statutory regulation or exceeds the permitted use, you will need to obtain permission directly from the copyright holder. To view a copy of this licence, visit http://creativecommonshorg/licenses/by/4. $0 /$.

\section{References}

1. AEDGE Collaboration, El-Neaj, Y.A., et al.: AEDGE: Atomic experiment for dark matter and gravity exploration in space. EPJ Quant. Technol. 7, 6 (2020). arXiv:1908.00802 
2. CERN: Workshop on atomic experiments for dark matter and gravity exploration. https://indico.cern. ch/event/830432/ (2019)

3. Planck Collaboration, Aghanim, N., et al.: Planck 2018 results. VI. Cosmological parameters, arXiv:1807.06209 (2018)

4. LIGO Scientific Collaboration, Aasi, J., et al.: Advanced LIGO. Class. Quant. Grav. 32, 074001 (2015). arXiv:1411.4547

5. VIRGO Collaboration, Acernese, F., et al.: Advanced Virgo: a second-generation interferometric gravitational wave detector. Class. Quant. Grav. 32, 024001 (2015). arXiv:1408.3978

6. KAGRA Collaboration, Somiya, K.: Detector configuration of KAGRA: The Japanese cryogenic gravitational-wave detector. Class. Quant. Grav. 29, 124007 (2012). arXiv:1111.7185

7. Unnikrishnan, C.S.: IndIGO and LIGO-India: Scope and plans for gravitational wave research and precision metrology in India. Int. J. Mod. Phys. D22, 1341010 (2013). arXiv:1510.06059

8. Punturo, M., et al.: The Einstein Telescope: A third-generation gravitational wave observatory. Class. Quant. Grav. 27, 194002 (2010)

9. Sathyaprakash, B., et al.: Scientific objectives of einstein telescope. Class. Quant. Grav. 29, 124013 (2012). arXiv:1206.0331

10. Reitze, D., et al.: Cosmic Explorer: The U.S. Contribution to Gravitational-Wave Astronomy beyond LIGO. Bull. Am. Astron. Soc. 51, 035 (2019). arXiv:1907.04833

11. Ruan, W.-H., Guo, Z.-K., Cai, R.-G., Zhang, Y.-Z.: Taiji program: Gravitational-wave sources. Int. J. Mod. Phys. A 35, 2050075 (2020). arXiv:1807.09495

12. Luo, J., Chen, L.-S., Duan, H.-Z., Gong, Y.-G., Hu, S., Ji, J., et al.: TianQin: A space-borne gravitational wave detector. Classic. Quantum Grav. 33, 035010 (2016)

13. Bender, P.L., Begelman, M.C., Gair, J.R.: Possible LISA follow-on mission scientific objectives. Class. Quant. Grav. 30, 165017 (2013)

14. Kawamura, S., et al.: The Japanese space gravitational wave antenna: DECIGO. Class. Quant. Grav. 28, 094011 (2011)

15. Mandel, I., Sesana, A., Vecchio, A.: The astrophysical science case for a decihertz gravitational-wave detector. Class. Quant. Grav. 35, 054004 (2018). arXiv:1710.11187

16. Baker, J., et al.: Space Based Gravitational Wave Astronomy Beyond LISA (Astro2020 APC White Paper), arXiv:1907.11305 (2019)

17. Kuns, K.A., Yu, H., Chen, Y., Adhikari, R.X.: Astrophysics and cosmology with a decihertz gravitational-wave detector: TianGO. Phys. Rev. D 102, 043001 (2020). arXiv:1908.06004

18. Pezzè, L., Smerzi, A., Oberthaler, M.K., Schmied, R., Treutlein, P.: Quantum metrology with nonclassical states of atomic ensembles. Rev. Mod. Phys. 90, 035005 (2018). arXiv:1609.01609

19. Graham, P.W., Hogan, J.M., Kasevich, M.A., Rajendran, S.: A new method for gravitational wave detection with atomic sensors. Phys. Rev. Lett. 110, 171102 (2013). arXiv:1206.0818

20. Graham, P.W., Hogan, J.M., Kasevich, M.A., Rajendran, S.: Resonant mode for gravitational wave detectors based on atom interferometry. Phys. Rev. D94, 104022 (2016). arXiv:1606.01860

21. MAGIS Collaboration, Graham, P.W., Hogan, J.M., Kasevich, M.A., Rajendran, S., Romani, R.W.: Mid-band gravitational wave detection with precision atomic sensors. arXiv:1711.02225 (2017)

22. Tino, G.M., et al.: SAGE: A proposal for a space atomic gravity explorer Eur. Phys. J. D Topical Issue on Quantum Technologies for Gravitational Physics. Eur. Phys. J. D 73, 228 (2019). arXiv:1907.03867

23. Canuel, B., et al.: Exploring gravity with the MIGA large scale atom interferometer. Sci. Rep. 8, 14064 (2018). arXiv:1703.02490

24. Zhan, M.-S., et al.: ZAIGA: Zhaoshan long-baseline atom interferometer gravitation antenna. Int. J. Mod. Phys. D28, 1940005 (2019). arXiv:1903.09288

25. Badurina, L., et al.: AION: An atom interferometer observatory and network. JCAP 05, 011 (2020). arXiv:1911.11755

26. Rosi, G., Sorrentino, F., Cacciapuoti, L., Prevedelli, M., Tino, G.M.: Precision measurement of the newtonian gravitational constant using cold atoms. Nature 510, 518 (2014). arXiv:1412.7954

27. Tino, G.M., et al.: Ultracold atoms and precision measurements. http://coldatoms.lens.unifi.it (2016)

28. ELGAR collaboration, Sabulsky, D.: The European Laboratory for Gravitation and Atom- interferometric Research (ELGAR) Project https://indico.cern.ch/event/830432/contributions/3497166/ attachments/1883894/3104651/sabulsky_ELGAR_CERN_2019.pdf (2019)

29. Liu, L., Lü, D.-S., Chen, W.-B., Li, T., Qu, Q.-Z., Wang, B., et al.: In-orbit operation of an atomic clock based on laser-cooled 87Rb atoms. Nature Communications 1-8 (2018) 
30. Becker, D. et al.: Space-borne Bose-einstein condensation for precision interferometry. Nature 562, 391 (2018)

31. Elliott, E.R., Krutzik, M.C., Williams, J.R., Thompson, R.J., Aveline, D.C.: Nasa's cold atom lab (cal): system development and ground test status. npj Microgravity 4, 16 (2016)

32. Lezius, M., et al.: Space-borne frequency comb metrology. Optica 3, 1381 (2016)

33. Dinkelaker, A., et al.: Space-borne frequency comb metrology. Appl. Opt. 56, 1388 (2017)

34. Döringshoff, K., et al.: Iodine frequency reference on a sounding rocket. Phys. Rev. Appl. 11, 054068 (2019)

35. Cacciapuoti, L., Salomon, C.: The ACES experiment. European Phys. J. Special Topics 172, 57 (2009)

36. Laurent, P., Massonnet, D., Cacciapuoti, L., Salomon, C.: The ACES/PHARAO space mission. Comptes Rendus Physique 16(5), 540 (2015)

37. BECCAL collaboration, Becker, D., et al.: BECCAL science overview. https://custom.cvent.com/ 216E523D934443CA9F514B796474A210/files/f7a0cce2d06f4e2182eaec7af912d5bf.pdf (2019)

38. Condon, G., et al.: All-Optical Bose-einstein condensates in microgravity. Phys. Rev. Lett. 123, 240402 (2019). arXiv:1906.10063

39. Bongs, K. et al.: Development of a strontium optical lattice clock for the SOC mission on the ISS. Comptes Rendus Physique 16(5), 553 (2015)

40. Origlia, S., et al.: Development of a strontium optical lattice clock for the SOC mission on the ISS. Phys. Rev. A 98, 053443 (2015)

41. Aguilera, D., et al.: STE-QUEST - Test of the universality of free fall using cold atom interferometry. Class. Quant. Grav. 31, 115010 (2014). arXiv:1312.5980

42. Lacour, S., et al.: SAGE: finding IMBH in the black hole desert. Class. Quant. Grav. 36, 195005 (2019). arXiv: 1811.04743

43. Gao, D., Wang, J., Zhan, M.: Atomic interferometric Gravitational-Wave space observatory (AIGSO). Commun. Theor. Phys. 69, 37 (2018)

44. Ellis, J., Vaskonen, V.: Probes of gravitational waves with atom interferometers. Phys. Rev. D 101, 12 (2020). arXiv:2003.13480

45. Aveline, D. et al.: Observation of Bose-Einstein condensates in an Earth-orbiting research lab. Nature 582, 193-197 (2020)

46. Yin, J. et al.: Entanglement-based secure quantum cryptography over 1,120 kilometres. Nature 582, 501-505 (2020)

Publisher's note Springer Nature remains neutral with regard to jurisdictional claims in published maps and institutional affiliations. 


\section{Affiliations}

Andrea Bertoldi ${ }^{1} \cdot$ Kai Bongs $^{2} \cdot$ Philippe Bouyer $^{1}$. Oliver Buchmueller ${ }^{3}$ (i) . Benjamin Canuel $^{1}$ - Laurentiu-loan Caramete ${ }^{4}$. Maria Luisa Chiofalo ${ }^{5}$. Jonathon Coleman ${ }^{6}$. Albert De Roeck ${ }^{7,8}$. John Ellis ${ }^{9,10,11}$. Peter W. Graham ${ }^{12}$. Martin G. Haehnelt ${ }^{13}$. Aurélien Hees ${ }^{14}$. Jason Hogan ${ }^{12}$. Wolf von Klitzing ${ }^{15}$. Markus Krutzik $^{16}$. Marek Lewicki ${ }^{9,17}$. Christopher McCabe ${ }^{9}$. Achim Peters ${ }^{16}$. Ernst Rasel $^{18}$. Albert Roura ${ }^{19}$. Dylan Sabulsky ${ }^{1}$. Stephan Schiller ${ }^{20}$. Christian Schubert ${ }^{18}$. Carla Signorini ${ }^{5}$. Fiodor Sorrentino ${ }^{21}$. Yeshpal Singh ${ }^{2}$. Guglielmo Maria Tino ${ }^{22,23}$. Ville Vaskonen ${ }^{9,10}$. Ming-Sheng Zhan ${ }^{24}$

1 LP2N, Laboratoire Photonique, Numérique et Nanosciences, Université Bordeaux-IOGS-CNRS:UMR 5298, F-33400, Talence, France

2 Department of Physics and Astronomy, University of Birmingham, Edgbaston, Birmingham, B15 2TT, UK

3 High Energy Physics Group, Blackett Laboratory, Imperial College, Prince Consort Road, London, SW7 2AZ, UK

4 Institute of Space Science, 409, Atomistilor Street Magurele, 077125, Ilfov, Romania

5 Dipartimento di Fisica "Enrico Fermi”, Universià di Pisa and INFN, Largo Bruno Pontecorvo 3, I-56126, Pisa, Italy

6 University of Liverpool, Liverpool, L69 7ZE, UK

7 Antwerp University, B 2610, Wilrijk, Belgium

8 Experimental Physics Department, CERN, CH-1211 Geneva 23, Geneva, Switzerland

9 Department of Physics, King's College London, Strand, WC2R 2LS, London, UK

10 National Institute of Chemical Physics \& Biophysics, Rävala 10, 10143, Tallinn, Estonia

11 Theoretical Physics Department, CERN, CH-1211 Geneva 23, Geneva, Switzerland

12 Department of Physics, Stanford University, Stanford, CA, 94305, USA

13 Kavli Institute for Cosmology and Institute of Astronomy, Madingley Road, Cambridge, CB3 0HA, UK

14 SYRTE, Observatoire de Paris, Université PSL, CNRS, Sorbonne Univesité, LNE, 61 avenue de l'Observatoire, 75014, Paris, France

15 Institute of Electronic Structure and Laser, Foundation for Research and Technology-Hellas, Heraklion, 70013, Greece

16 Humboldt Universität zu Berlin, Institute of Physics, Newtonstrasse 15, 12489, Berlin, Germany

17 Faculty of Physics, University of Warsaw, ul. Pasteura 5, 02-093, Warsaw, Poland

18 Institut fuür Quantenoptik, Leibniz Universität Hannover, Welfengarten 1, D-30167, Hannover, Germany

19 Institute of Quantum Technologies, German Aerospace Center (DLR), Söflinger Str. 100, 89077, Ulm, Germany

20 Institut für Experimentalphysik, Heinrich-Heine-Universität Düsseldorf, Düsseldorf, Germany

21 Istituto Nazionale di Fisica Nucleare, Sez. di Genova, via Dodecaneso 33, Genova, Italy

22 Dipartimento di Fisica e Astronomia and LENS, Università di Firenze, Firenze, Italy

23 Istituto Nazionale di Fisica Nucleare, via Sansone 1, I-50019, Sesto Fiorentino, Firenze, Italy

24 State Key Laboratory of Magnetic Resonance and Atomic and Molecular Physics, Wuhan Institute of Physics and Mathematics, Chinese Academy of Sciences, Wuhan, 430071, China 